\title{
The prevalence of albuminuria among diabetic patients in a primary care setting in Singapore
}

\author{
Eng Sing Lee ${ }^{1}$, MBChB, MMed, Wern Ee $\underline{\text { Tang }^{1}}$, MBBS, MMed
}

\begin{abstract}
INTRODUCTION Microalbuminuria is an early sign of kidney damage. The prevalence of microalbuminuria in Singapore has been reported to be $36.0 \%-48.5 \%$. However, the prevalence of microalbuminuria reported in these studies was determined with one urine sample using a qualitative urine test. The aim of this study was to determine the prevalence of micro- and macroalbuminuria using a more stringent criterion of two positive quantitative urine albumin-creatinine ratio (ACR) tests. METHODS We conducted a cross-sectional study of patients with type 2 diabetes mellitus (T2DM) who were followed up at a primary care clinic in Singapore. Patients were diagnosed to have albuminuria if they had two positive ACR tests within a seven-month period.

RESULTS A total of 786 patients with T2DM met the study's inclusion criteria. $55.7 \%$ were already on an angiotensin-converting enzyme inhibitor (ACEI) and/or angiotensin receptor blocker (ARB). The prevalence rates of micro- and macroalbuminuria were $14.2 \%$ and $5.7 \%$, respectively. Patients with albuminuria were more likely to have hypertension (odds ratio [OR] 3.47 , 95\% confidence interval [ $\mathrm{Cl}] 1.55-7.80)$. Diabetics with poorer diabetic control (OR $1.88,95 \% \mathrm{Cl} 1.26-2.79)$, and higher systolic (OR 1.69, 95\% Cl 1.14-2.49) and diastolic (OR 1.96, 95\% Cl, 1.20 to 3.22) blood pressures were more likely to have albuminuria.

CONCLUSION In the present study, the prevalence of microalbuminuria is significantly lower than that previously reported in Singapore. The presence of hypertension, poor diabetic control and suboptimal blood pressure control are possible risk factors for albuminuria in patients with T2DM.
\end{abstract}

Keywords: albuminuria, macroalbuminuria, microalbuminuria, prevalence, type 2 diabetes mellitus

\section{INTRODUCTION}

Diabetes mellitus is associated with multiple pathologies, including nephropathy. Around $5 \%-10 \%$ of type 2 diabetes mellitus (T2DM) patients with microalbuminuria develop diabetic nephropathy annually. ${ }^{(1)}$ The latest Singapore Renal Registry Statistics shows that, as an aetiology, diabetic nephropathy accounts for $64.5 \%$ of end stage renal failure. ${ }^{(2)}$ Proteinuria is strongly correlated to progression of renal failure, and has been shown to be a 'nephrotoxin'. ${ }^{(3)}$

Microalbuminuria in diabetic patients has been recognised as not only a predictor of progression of diabetic nephropathy, but also a powerful independent risk factor for cardiovascular disease. ${ }^{(4)}$ Annual screening of microalbuminuria is recommended for all diabetic patients. Many studies have confirmed that an angiotensin-converting enzyme inhibitor (ACEI) or angiotensin receptor blocker (ARB) can retard the progression of albumin excretion. However, multiple factors influence the appearance of albumin in urine. ${ }^{(5)}$ These include ketosis, hyperglycaemia, physical exercise, dietary protein intake, diuresis and the presence of urinary tract infection. Inter-individual variability can be as high as $40 \%$. Due to this variability, confirmation of albumin excretion with additional tests during the subsequent 3-6 months is recommended.

Semi-quantitative tests such as urine test strips for microalbuminuria (the micral test) were used by the National Healthcare Group Polyclinics (NHGP) until mid-2010. The micral test, which gives a qualitative urine test result of normo-, micro- or macroalbuminuria, is suitable as a screening test but not for diagnosis. ${ }^{(6)}$ When following up patients with microalbuminuria, the micral test is also not helpful if the quantity of secreted albumin is unknown. Thus, any measures to delay or curb the disease progression will not be objectively known. The micral test has been replaced by the quantitative urine albumin-creatinine ratio (ACR) test in all NHGP institutions since July 2010. The ACR test is more accurate, as spot creatinine helps to minimise errors caused by concentrated or diluted urine. The ACR test has also been found to accurately correlate with 24-hour urinary protein collection. ${ }^{(7)}$

Multiple studies conducted overseas have demonstrated that the prevalence of microalbuminuria varies among races, even within the same community. ${ }^{(8)}$ As such, data extrapolated from overseas studies would not apply to our local population. Three local studies on the prevalence of microalbuminuria in Singapore have been published, ${ }^{(9-11)}$ with reported prevalence rates ranging from $36.0 \%$ to $48.5 \%$. However, the majority of these studies were based on semi-quantitative tests with only a single urine sample collected.

The present study aimed to establish the current baseline prevalence of micro- and macroalbuminuria in Singapore using the ACR test. This is the first study in Singapore that used a quantitative method with at least two consecutive urine samples to determine the prevalence of micro- and macroalbuminuria in patients with T2DM. The main objective is to test the hypothesis that the prevalence of microalbuminuria was overestimated in 
previous studies conducted in Singapore. The secondary objective is to identify the possible predictors of albuminuria in patients with T2DM.

\section{METHODS}

This was a cross-sectional study conducted on patients with T2DM who attended Clementi Polyclinic, a public institution for primary care in Singapore. The study assessed the prevalence of micro- and macroalbuminuria in consecutively screened adult T2DM patients at Clementi Polyclinic over a seven-month period from 1 August 2010 to 28 February 2011. The diagnosis of T2DM was retrieved from the data recorded in the patient case notes. This study was approved by the Ethics Committee of NHG.

All diabetic patients who visit the polyclinic are offered an annual diabetes monitoring panel. The panel consists of biochemical analyses of serum and urine, including glycated haemoglobin (HbA1c) test, lipid profile, urine dipstick, urine microalbumin, fasting glucose, serum creatinine, sodium and potassium. The urine microalbumin test, i.e. ACR, (Advia 2400, Siemens Healthcare Diagnostics, Erlangen, Germany) is a quantitative test conducted via a random spot urine specimen. All urine microalbumin tests are conducted in the morning to eliminate the effect of orthostatic proteinuria. ${ }^{(12)}$ The proteincreatinine ratio (PCR) test may be used in place of the ACR test if ACR is more than $30 \mathrm{mg} / \mathrm{mmoL}$; this is because the relationship between ACR and PCR is non-linear, and higher ACRs could not adequately predict the amount of 24-hour urinary protein. ${ }^{(13)}$ Since the switch from the micral test to the quantitative urine ACR test for diagnosis of microalbuminuria, all doctors working in the polyclinic had been briefed and reminded to offer a second urine test if the first urine sample showed ACR $>2.5 \mathrm{mg} / \mathrm{mmoL}$ for male patients and $>3.5 \mathrm{mg} / \mathrm{mmoL}$ for female patients.

T2DM patients aged $>18$ years who underwent the diabetes monitoring panel from 1 August 2010 to 31 October 2010 were selected for the present study. Patients who were detected to have micro- or macroalbuminuria during that period were followed up till 28 February 2011 (up to four months) and underwent a second urine test. The exclusion criteria were: (a) patients with leucocytes $\geq 1+$ or positive nitrite detected in the urine dipstick test (both were indicative of bacteriuria); (b) patients whose urine sample showed red blood cells $\geq 1+$; (c) patients with type 1 diabetes mellitus (T1DM) who had known proteinuria or chronic kidney disease (CKD) stage 3 and beyond (i.e. glomerular filtration rate $<60 \mathrm{~mL} / \mathrm{min} / 1.72 \mathrm{~m}^{2}$ ), prior to the diagnosis of T1DM; and (d) patients who did not have a second urine test when indicated. The electronic medical notes and a computerised patient support system were used to identify patients who met the inclusion criteria, as well as to assess the relevant history, laboratory investigation reports and prescriptions of each identified patient.

Normoalbuminuria was defined as urinary ACR $\leq 2.5 \mathrm{mg} / \mathrm{mmoL}$ for male and ACR $\leq 3.5 \mathrm{mg} / \mathrm{mmoL}$ for female patients. Patients who were found to have normoalbuminuria at the first urine test were not offered a second urine test. Male and female patients whose first urine sample showed ACR $>2.5 \mathrm{mg} / \mathrm{mmoL}$ and ACR $>3.5 \mathrm{mg} / \mathrm{mmoL}$, respectively, but whose second urine sample
Table I. A summary of the definitions of albuminuria. ${ }^{(13)}$

\begin{tabular}{llc}
\hline Characteristic & ACR $(\mathbf{m g} / \mathbf{m m o L})$ & PCR $(\mathbf{m g} / \mathbf{m m o L})$ \\
\hline Normoalbuminuria & $\leq 2.5$ (male) & $\leq 19$ \\
& $\leq 3.5$ (female) & \\
Microalbuminuria & $>2.5$ to 30 (male) & $>19$ to 50 \\
Macroalbuminuria & $>30.5$ to 30 (female) & \\
\hline
\end{tabular}

ACR: albumin-creatinine ratio; PCR: protein-creatinine ratio

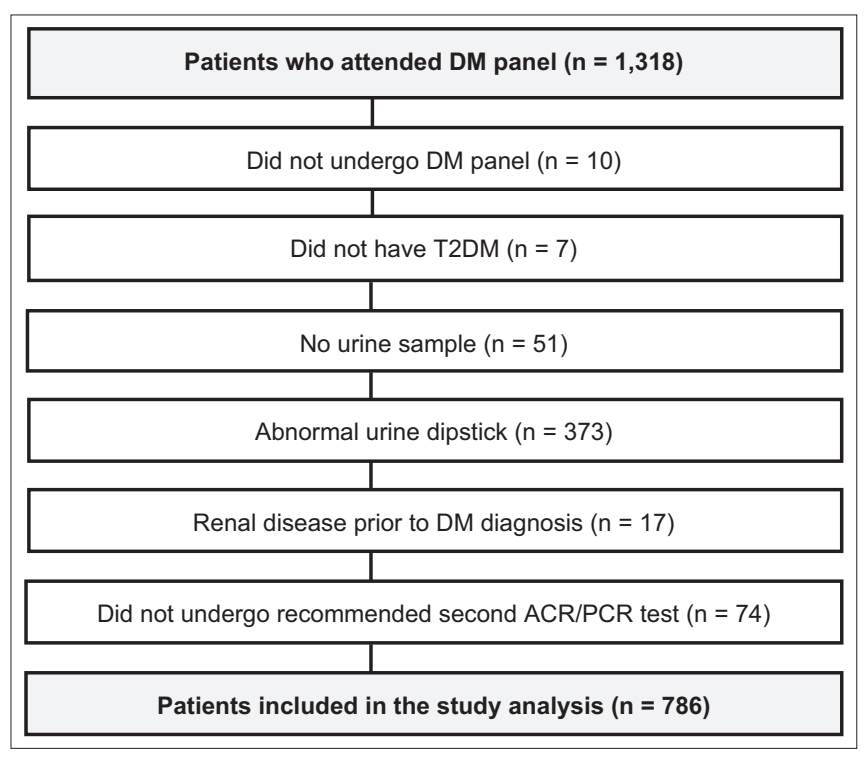

Fig. 1 Flowchart shows the process of sample selection. ACR: albumincreatinine ratio; DM: diabetes mellitus; PCR: protein-creatinine ratio; T2DM: type 2 diabetes mellitus

showed ACR less than the cutoff for each gender were deemed to have normoalbuminuria. Patients were classified as having microalbuminuria if both urine samples showed ACRs from $>2.5 \mathrm{mg} / \mathrm{mmoL}$ to $30 \mathrm{mg} / \mathrm{mmoL}$ for male and from $>3.5 \mathrm{mg} / \mathrm{mmoL}$ to $30 \mathrm{mg} / \mathrm{mmoL}$ for female patients, or PCR $>19 \mathrm{mg} / \mathrm{mmoL}$ to $50 \mathrm{mg} / \mathrm{mmoL}$ for both genders. Patients who were found to have macroalbuminuria at the first urine test but subsequently developed microalbuminuria (detected at the second urine test) were deemed to have microalbuminuria. If both the urine samples showed ACR > $30 \mathrm{mg} / \mathrm{mmoL}$ or PCR $>50 \mathrm{mg} / \mathrm{mmoL}$, the patient was classified as having macroalbuminuria. Patients who were found to have microalbuminuria at the first urine test but who subsequently developed macroalbuminuria (detected at the second urine test) were classified as having macroalbuminuria (Table I).

The sample size for the present trial was calculated based on the assumption that the prevalence of microalbuminuria is less than $30 \%$, which is lower than the lowest prevalence of $36 \%$ observed in the three local studies. ${ }^{(9-11)}$ Computing from a type I error of $1 \%$ and a type II error of $20 \%$, at least 672 patients were required for the trial analysis. After including an overall dropout rate of $30 \%$ (i.e. patients who do not return for their second urine test), the trial would require a total enrolment of 874 patients. On the average, 10-20 patients per day undergo diabetes monitoring panel tests at the polyclinic. Therefore, it took 13 weeks (from 1 August 2010 to 31 October 2010) to 
Table II. Patient characteristics by albuminuria level.

\begin{tabular}{|c|c|c|c|c|c|}
\hline Characteristic & All & Normoalbuminuria & Microalbuminuria & Macroalbuminuria & p-value \\
\hline No. (\%) & $786(100)$ & $629(80.0)$ & $112(14.2)$ & $45(5.7)$ & - \\
\hline Mean age* (yr) & $63.95(10.36)$ & $63.09(10.16)$ & $66.55(10.44)$ & $69.40(10.43)$ & $<0.001^{\S}$ \\
\hline Gender & & & & & $0.007^{\S}$ \\
\hline Male (\%) & $462(58.8)$ & $356(56.6)$ & $81(72.3)$ & $25(55.6)$ & \\
\hline Mean DM duration* (yr) & $7.04(5.16)$ & $6.61(4.79)$ & $8.73(6.60)$ & $8.80(4.84)$ & $<0.001^{\text {s }}$ \\
\hline Median DM duration+ (yr) & $6.78(4.52)$ & $6.34(4.95)$ & $7.86(4.60)$ & $8.19(2.90)$ & $<0.001^{\mathrm{s}}$ \\
\hline Race & & & & & $0.017^{\ddagger}$ \\
\hline Chinese (\%) & $571(72.6)$ & $450(71.5)$ & $91(81.3)$ & $30(66.7)$ & \\
\hline Malay (\%) & $112(14.2)$ & $88(14.0)$ & $11(9.8)$ & $13(28.9)$ & \\
\hline Indian (\%) & $68(8.7)$ & $59(9.4)$ & $8(7.1)$ & $1(2.2)$ & \\
\hline \multicolumn{6}{|l|}{ Hypertension status } \\
\hline Yes (\%) & $653(83.1)$ & $503(80.0)$ & 105 (93.8) & $45(100.0)$ & $<0.001^{\mathrm{s}}$ \\
\hline ACEI (\%) & $332(50.8)$ & $257(51.1)$ & $49(46.7)$ & $26(57.8)$ & 0.447 \\
\hline ARB (\%) & $104(15.9)$ & $68(13.5)$ & $26(24.8)$ & $10(22.2)$ & $0.008^{\S}$ \\
\hline Total" (\%) & $425(65.1)$ & $317(63.0)$ & $72(68.6)$ & $36(80.0)$ & 0.052 \\
\hline No $(\%)$ & $133(16.9)$ & $126(20.0)$ & $7(6.3)$ & 0 & - \\
\hline ACEI (\%) & $12(9.0)$ & $10(7.9)$ & $2(28.6)$ & 0 & 0.064 \\
\hline ARB (\%) & $2(1.5)$ & $2(1.6)$ & 0 & 0 & 0.737 \\
\hline Total" (\%) & $13(9.8)$ & $11(8.7)$ & $2(28.6)$ & 0 & 0.085 \\
\hline HT duration* (yr) & $7.45(4.90)$ & $7.04(4.10)$ & $8.70(7.07)$ & $9.16(6.07)$ & $0.041^{*}$ \\
\hline Systolic BP+ (mmHg) & $126.5(19)$ & $125(19)$ & $130(20)$ & $138(23)$ & $<0.001^{\S}$ \\
\hline Diastolic $\mathrm{BP}^{+}(\mathrm{mmHg})$ & $70(13)$ & $69(13)$ & $70(15)$ & $70(15)$ & 0.060 \\
\hline $\mathrm{GFR}^{+}(\mathrm{mL} / \mathrm{min})$ & $84.44(29.12)$ & $86.24(29.02)$ & $79.09(25.51)$ & $67.86(38.31)$ & $<0.001^{\text {s }}$ \\
\hline $\mathrm{TC}^{+}(\mathrm{mmol} / \mathrm{L})$ & $4.32(0.95)$ & $4.32(0.95)$ & $4.28(0.96)$ & $4.38(0.92)$ & 0.591 \\
\hline $\mathrm{LDL}^{+}(\mathrm{mmol} / \mathrm{L})$ & $2.40(0.75)$ & $2.43(0.75)$ & $2.36(0.79)$ & $2.33(0.80)$ & 0.367 \\
\hline $\mathrm{HDL}^{+}(\mathrm{mmol} / \mathrm{L})$ & $1.21(0.37)$ & $1.22(0.36)$ & $1.15(0.30)$ & $1.18(0.56)$ & 0.157 \\
\hline $\mathrm{TG}^{+}(\mathrm{mmol} / \mathrm{L})$ & $1.33(0.80)$ & $1.32(0.79)$ & $1.35(0.91)$ & $1.40(0.72)$ & 0.296 \\
\hline
\end{tabular}

Overall, age was normally distributed (tested by Shapiro-Wilk test with $\mathrm{p}$-value $=0.131$ ). However, the age of the normoalbuminuria group was not normally distributed $(p=0.017)$. * Data presented as mean (standard deviation). †Data presented as median (interquartile range). A $p$-value $¥<0.05 ; \S<0.01$ indicated statistical significance. "Total number of patients on angiotensin-converting enzyme inhibitors (ACEI) and/or angiotensin receptor blockers (ARB). BP: blood pressure; DM: diabetes mellitus; GFR: glomerular filtration rate; HbA1c: glycated haemoglobin; HDL: high density lipids; HT: hypertension; LDL: low density lipids; TC: total cholesterol; TG: triglycerides

recruit the required number of patients (15 patients $\times 6$ days/ week $\times 13$ weeks $=1,170$ patients). Analysis of the data was done using IBM SPSS Statistics version 19.0 (IBM Corp, Armonk, NY, USA). Descriptive statistics and multivariate logistic regression tests were used.

\section{RESULTS}

A total of 1,318 patients attended diabetes monitoring panel tests at Clementi Polyclinic during the described time frame. 216 patients required a second confirmatory urine test. Of the 216 patients, 74 did not turn up for their second ACR or PCR tests. Finally, 786 eligible patients were included in the study analysis (Fig. 1).

The overall prevalence of micro- and macroalbuminuria among the T2DM patients was $14.2 \%$ and $5.7 \%$, respectively (Table II). About $19.0 \%$ of the patients who initially had microalbuminuria (29 out of 153 ) reverted to normoalbuminuria with a repeat urine test. However, no patients with initial macroalbuminuria were reclassified to normoalbuminuria with the repeat urine test. Following the reclassification, the prevalence of normoalbuminuria increased from $76.3 \%$ to $80.0 \%$.

Among those who were found to have macroalbuminuria at the first urine test, $18.2 \%$ ( 6 out of 33 ) were reclassified as having microalbuminuria with the repeat urine test. $69.3 \%$ of patients (106 out of 153) who were classified with microalbuminuria remained in the same category after the second urine test. $11.8 \%$ of patients (18 out of 153) who were initially found to have microalbuminuria were reclassified as having macroalbuminuria after the second urine test. The second urine ACR test reduced the prevalence of microalbuminuria from $19.5 \%$ to $14.2 \%$. At the first urine test, 33 patients were found to have macroalbuminuria, but the total number increased to 45 after the second urine test. $81.8 \%$ of patients (27 out of 33 ) who were classified as having macroalbuminuria remained in the same category after the second urine test. The prevalence of macroalbuminuria increased from $4.2 \%$ to $5.7 \%$ after the reclassification. 
Multivariate logistic regression analysis was performed to study the potential predictors of albuminuria (microalbuminuria and macroalbuminuria). The odds of developing albuminuria among hypertensive T2DM patients were about 3.5 times as high as that among non-hypertensive T2DM patients (adjusted odds ratio [OR] 3.47, 95\% confidence interval [CI] 1.55-7.80). The odds of developing albuminuria among T2DM patients with glomerular filtration rate $(\mathrm{GFR})<60 \mathrm{~mL} / \mathrm{min}$ (i.e. CKD stage 3 and beyond) were two times as high as that of T2DM patients with normal GFR (adjusted OR 2.14, 95\% Cl 1.36-3.38). The odds of having albuminuria among T2DM patients with suboptimal DM control of $\mathrm{HbA} 1 \mathrm{c}>7.0 \%$ were $88 \%$ higher than those with $\mathrm{HbA} 1 \mathrm{c}$ $\leq 7.0 \%$ (adjusted OR 1.88, 95\% Cl 1.26-2.79). In addition, T2DM patients with the following characteristics have higher odds of being diagnosed with albuminuria: systolic blood pressure (BP) $\geq 130 \mathrm{mmHg}$ or diastolic $\mathrm{BP} \geq 80 \mathrm{mmHg}$; duration of T2DM $\geq$ 7 years; and male gender (Table III).

\section{DISCUSSION}

We compared the present study with three studies conducted in the local context, namely the Microalbuminuria Prevalence Study (MAPS), ${ }^{(9)}$ Singapore Prospective Study Programme ${ }^{(10)}$ and Diabcare study. ${ }^{(11)}$

In MAPS (Singapore), Wu et al ${ }^{(9)}$ sampled 388 patients using one sample of early morning urine. All the patients had hypertension (compared to $83.1 \%$ in the current study) and T2DM, with an average disease duration of 8.6 years, an average $\mathrm{HbA} 1 \mathrm{c}$ level of $7.9 \%$ and an average age of 58.3 years (about five years younger than the average age in the current study). The proportion of male to female participants was not reported. ACEI and ARB were only used in $34 \%$ and $23 \%$ of patients, respectively, ${ }^{(9)}$ as compared to $50.8 \%$ and $15.9 \%$ in the current study. The reported prevalence rates of micro- and macroalbuminuria were $48.5 \%$ and $23.5 \%$, respectively. Nang et al's study, ${ }^{(10)}$ which sampled 446 patients using one early morning urine sample, was a community-based study where the diabetic condition was self-reported. The prevalence of albuminuria was $37.6 \%$. The Diabcare study by Lee et $\mathrm{al}^{(11)}$ sampled 174 patients using one urine sample; no mention was made of whether they were early morning or random urine collections. The reported prevalence of albuminuria was 39\% (36\% microalbuminuria and 3\% macroalbuminuria). $91.4 \%$ of the patients had T2DM, 8.2\% had T1DM and $0.4 \%$ were classified as others; $22.8 \%$ of the study population were hypertensive. The average age and duration of DM were 58.1 years and 10.1 years, respectively. The gender distribution was $52.3 \%$ female and $48.1 \%$ male. ${ }^{(11)}$

Comparing the demographics of the current study with that of the aforementioned studies, our study comprised participants who were generally older and a higher proportion of males. The racial proportion of this study is comparable to that of the studies of Nang et $\mathrm{al}^{\left({ }^{(10)}\right.}$ and Lee et al. ${ }^{(11)}$ The final prevalence rates of micro- and macroalbuminuria in the current study were $14.2 \%$ and $5.7 \%$, respectively; these figures are much lower than the rates of $48.5 \%$ and $23.5 \%$ reported in MAPS (Singapore). ${ }^{(9)}$ Possible reasons for this large disparity included: (a) difference in BP measurements
Table III. Predictors of proteinuria in patients with type 2 diabetes mellitus (T2DM) from a logistic regression model.

\begin{tabular}{|c|c|c|}
\hline Covariates & Adjusted OR (95\% CI) & p-value \\
\hline Duration of DM (yr) & & 0.001 \\
\hline$<7$ & 1.00 (ref) & \\
\hline$\geq 7$ & $1.87(1.27-2.74)$ & \\
\hline Gender & & 0.022 \\
\hline Female & 1.00 (ref) & \\
\hline Male & $1.58(1.07-2.33)$ & \\
\hline Hypertension status & & 0.003 \\
\hline No & 1.00 (ref) & \\
\hline Yes & $3.47(1.55-7.80)$ & \\
\hline Systolic BP (mmHg) & & 0.008 \\
\hline$<130$ & 1.00 (ref) & \\
\hline$\geq 130$ & $1.69(1.14-2.49)$ & \\
\hline Diastolic BP (mmHg) & & 0.007 \\
\hline$<80$ & 1.00 (ref) & \\
\hline$\geq 80$ & $1.96(1.20-3.22)$ & \\
\hline HbA1c (\%) & & 0.002 \\
\hline$\leq 7.0$ & 1.00 (ref) & \\
\hline$>7.0$ & $1.88(1.26-2.79)$ & \\
\hline GFR (mL/min) & & 0.001 \\
\hline$\geq 60$ & 1.00 (ref) & \\
\hline$<60$ & $2.14(1.36-3.38)$ & \\
\hline
\end{tabular}

Non-proteinuria patients were used as a reference group (ref). BP: blood pressure; $\mathrm{Cl}$ : confidence interval; DM: diabetes mellitus; GFR: glomerular filtration rate; HbA1c: glycated haemoglobin; OR: odds ratio

(median BP in our study was $127 / 70 \mathrm{mmHg}$, while mean BP in MAPS [Singapore] was 144/84 mmHg); (b) the median HbA1c in our study was $0.7 \%$ lower than the mean reported in MAPS (Singapore); and (c) there was a lower proportion of patients who were on antiproteinuric agents in MAPS (Singapore). The $37.6 \%$ prevalence of proteinuria (i.e. micro and macro) reported in the Singapore Prospective Study Programme ${ }^{(10)}$ was also higher than the $19.9 \%$ prevalence $(14.2 \%$ micro and $5.7 \%$ macro) found in the current study.

Similarly, the prevalence of microalbuminuria in the current study was less than half of that reported in the Diabcare study (36\%). ${ }^{(11)}$ There could be several reasons for the lower prevalence found in our study. First, the quantitative method (i.e. ACR test) used in the current study is a more accurate way of determining albumin levels, whereas in previous studies, the use of semiquantitative dipstick tests to screen for microalbuminuria could have overestimated its prevalence. Second, up to two-thirds of the T2DM patients with albuminuria had been taking antiproteinuric agents prior to the current study. There is extensive and conclusive evidence that blockage of the renin-angiotensin-aldosterone system is a highly effective strategy to decrease the amount of albumin in the urine. ${ }^{(14)}$ Therefore, it is likely that the patients who were on antiproteinuric agents could have reverted to normoalbuminuria and were thus classified as such, reducing the prevalence of albuminuria in the current study. Finally, the methods used in this study may have reduced the prevalence rates, as only patients who had albuminuria were advised to return for a repeat urine test, whereas those with normoalbuminuria were 
not. Also, the second urine sample was preferred to determine a definitive classification, if the result was incongruent with the first sample. This criterion was used as the first ACR tests were done on fasting samples conducted together with the diabetes monitoring panel. The second urine sample was tested in a nonfasting state and therefore reduced the chances of dehydration, a possible cause of albuminuria. However, the impact of this reclassification method was small, as there were only slight changes in the prevalence of micro- (from $19.5 \%$ to $14.2 \%$ ) and macroalbuminuria (from $4.2 \%$ to $5.7 \%$ ).

In the current study, the prevalence of albuminuria (both micro and macro) after the reclassification was reduced from $23.7 \%$ to $19.9 \%$; even then, the higher pre-classification rate of $23.7 \%$ was still considerably lower than that reported by previous studies. Hence, a lower prevalence of microalbuminuria was found among T2DM patients in this study compared with previous studies conducted in Singapore. Similarly, a Minnesota study reported a $60 \%$ decline in the prevalence of proteinuria among people with adult-onset DM between 1970 and 1990, ${ }^{(15)}$ although the cause of decline over time was not known.

In terms of the usage of ACEI and ARB, the current study showed that ACEI (43.8\%) was used more frequently than ARB $(13.5 \%)$ in patients with T2DM. As ACEI and ARB are different classes of drugs, clinical equivalence cannot be assumed. Mechanistically speaking, ARB may be a better renin-angiotensinaldosterone-system blocking agent than ACEI. ${ }^{(16)}$ Moreover, a $20 \%-50 \%$ incidence of ACEl-associated coughing has been reported in Chinese patients. ${ }^{(17)}$ Whether ARB is more appropriate than ACEI for the treatment of diabetic nephropathy in Asians is an important clinical and economical consideration, especially in the public setting.

The potential predictors of albuminuria found in the current study included: (a) a diagnosis of hypertension; (b) actual BP $\geq 130 / 80 \mathrm{mmHg}$; (c) male T2DM patients; (d) HbA1c > 7.0\%; (e) duration of DM $\geq 7$ years; and (f) T2DM patients with CKD stage 3 and beyond. Interestingly, all the parameters of the lipid panel did not seem to have a significant impact on albuminuria. In the post-hoc analysis, T2DM patients of Malay ethnic origin seemed to have higher odds of developing macro- but not microalbuminuria.

This study is not without its limitations. First, the authors opine that, realistically, all patients would not undergo retesting of ACRs within one year if the result was normal, as recommended in NHG's institutional guidelines. ${ }^{(18)}$ This method of classifying albuminuria reduces costs and allows for easier comparison in future studies. Johnson et al ${ }^{(13)}$ likewise recommended that if the initial urine ACR test was negative, repeat testing was not required until the next recommended testing interval. However, the criteria for diagnosis of albuminuria in the current study did not strictly adhere to the Kidney Disease Outcomes Quality Initiative Clinical Practice Guidelines. ${ }^{(5)}$ Second, non-diabetic renal disease as an underlying cause of albuminuria ${ }^{(19)}$ was not excluded in the current study. Also, we did not explore or examine patients who have had their albuminuria successfully treated with ACEI or ARB and the genetic impact of polymorphism of the ACE gene on the progression of diabetic nephropathy. ${ }^{(20)}$ Finally, selection bias could not be excluded, as the study population might have included a higher proportion of motivated T2DM patients who attend their annual panel tests regularly and repeat urine tests when indicated.

In conclusion, the current study has established a baseline reference for the prevalence of micro- and macroalbuminuria among T2DM patients in a primary care setting in Singapore. Lower prevalence rates were found in this study as compared with other local studies. However, the better methodology used in this study could not fully account for the lower prevalence rates, and thus, the usage of ACEI and ARB may be an important contributing factor. This study also suggests that T2DM patients with coexisting hypertension have a higher prevalence of albuminuria. As T2DM patients tend to develop hypertension sooner than T1DM patients (raised BP being part of the metabolic syndrome) and there tends to be a higher number of T2DM patients, it will be prudent for primary care physicians to screen, control and treat diabetic nephropathy in T2DM patients early in the course of the disease. ${ }^{(21)}$ Future studies should be conducted to validate the methodology used in this study in order to confirm that the rigour of diagnosis will not be compromised by the practicability of clinical routine. Finally, future studies should also be designed to confirm the predictors of albuminuria, as suggested by this study.

\section{ACKNOWLEDGEMENTS}

We would like to thank Prof Su Chi Lim, Deputy Director, Diabetes Centre and Senior Consultant, Department of Medicine, Khoo Teck Puat Hospital, for his support and guidance, and Ms Yu Fan Sim, Executive, Clinical Research Unit, National Healthcare Group Polyclinics, for her assistance with the statistical analysis.

\section{REFERENCES}

1. Parving $\mathrm{HH}$, Lehnert $\mathrm{H}$, Bröchner-Mortensen J, et al; Irbesartan in Patients with Type 2 Diabetes and Microalbuminuria Study Group. The effect of irbesartan on the development of diabetic nephropathy in patients with type 2 diabetes. New Engl J Med 2001; 345:870-8.

2. Health Promotion Board. Singapore Renal Registry Annual Registry Report 1999 - 2013 (Preliminary). Available at: https:/www.nrdo.gov.sg/docs/ librariesprovider3/Publications---Kidney-Failure/singapore-renal-registryannual-registry-report-1999-2013-preliminary.pdf?sfvrsn=0. Accessed December 2, 2014.

3. Cravedi P, Remuzzi G. Pathophysiology of proteinuria and its value as an outcome measure in chronic kidney disease. Br J Clin Pharmacol 2013; 76:516-23.

4. Basi S, Fesler P, Mimran A, Lewis JB. Microalbuminuria in type 2 diabetes and hypertension: a marker, treatment target, or innocent bystander? Diabetes Care 2008; 31 Suppl 2:S194-201

5. KDOQI. KDOQI Clinical Practice Guidelines and Clinical Practice Recommendations for Diabetes and Chronic Kidney Disease. Am J Kidney Dis 2007; 49(2 Suppl 2):S12-154.

6. Leong SO, Lui KF, Ng WY, Thai AC. The use of semi-quantitative urine test-strip (Micral Test) for microalbuminuria screening in patients with diabetes mellitus. Singapore Med J 1998; 39:101-3.

7. Price CP, Newall RG, Boyd JC. Use of protein: creatinine ratio measurements on random urine samples for prediction of significant proteinuria: a systematic review. Clin Chem 2005; 51:1577-86.

8. Dreyer G, Hull S, Aitken Z, Chesser A, Yaqoob MM. The effect of ethnicity on the prevalence of diabetes and associated chronic kidney disease. QJM 2009; 102:261-9.

9. Wu AY, Tan $\mathrm{CB}$, Eng PH, et al. Microalbuminuria prevalence study in 
hypertensive patients with type 2 diabetes mellitus in Singapore. Singapore Med J 2006; 47:315-20.

10. Nang EE, Khoo CM, Tai ES, et al. Is there a clear threshold for fasting plasma glucose that differentiates between those with and without neuropathy and chronic kidney disease?: the Singapore Prospective Study Program. Am J Epidemiol 2009; 169:1454-62.

11. Lee WR, Lim HS, Thai AC, et al; Diabcare Singapore Local Working Group \& Diabetic Society of Singapore. A window on the current status of diabetes mellitus in Singapore--the Diabcare-Singapore 1998 study. Singapore Med J 2001; 42:501-7.

12. Martin-Du Pan RC, Benoit R, Girardier L. The role of body position and gravity in the symptoms and treatment of various medical diseases. Swiss Med Wkly 2004; 134:543-51.

13. Johnson DW, Jones GR, Mathew TH, et al; Australasian Proteinuria Consensus Working Group. Chronic kidney disease and measurement of albuminuria or proteinuria: a position statement. Med J Aust 2012; 197:224-5.

14. de Zeeuw D. Albuminuria: a target for treatment of type 2 diabetic nephropathy. Semin Nephrol 2007; 27:172-81.
15. Larson TS, Santanello N, Shahinfar S, et al. Trends in persistent proteinuria in adult-onset diabetes: a population-based study. Diabetes Care 2000; 23:51-6.

16. Lim SC, Koh AF, Goh SK, et al. Angiotensin receptor antagonist vs. angiotensin-converting enzyme inhibitor in Asian subjects with type 2 diabetes and albuminuria - a randomized crossover study. Diabetes Obes Metab 2007; 9:477-82.

17. Tomlinson B, Young RP, ChanJC, Chan TY, CritchleyJA. Pharmacoepidemiology of ACE inhibitor--induced cough. Drug Saf 1997; 16:150-1.

18. Lim CK. NUHS-NHGP Proteinuria Algorithm. Available at: http://www. pca.sg/files/plexus/2011/Plexus_Mar_2011.pdf. Accessed December 2, 2014.

19. Christensen PK, Larsen S, Horn T, Olsen S, Parving HH. Causes of albuminuria in patients with type 2 diabetes without diabetic retinopathy. Kidney Int 2000; 58:1719-31.

20. Jacobsen PK. Preventing end stage renal disease in diabetic patients-genetic aspect (part I). J Renin Angiotensin Aldosterone Syst 2005; 6:1-14.

21. Cooper ME, Jandeleit-Dahm K, Thomas MC. Targets to retard the progression of diabetic nephropathy. Kidney Int 2005; 68:1439-45. 